A C T A C H E M I C A S C A N D I N A V I C A 15 (1961)

\title{
The Solubility of Silver Chloride and Silver Bromide in Aqueous Ammonia and the Formation of Mixed Silver-Ammonia-Halide Complexes
}

\author{
I D O LEDEN* and G ÖRAN PERSSON \\ Department of Inorganic Chemistry, Chalmers Institute of Technology, Göteborg, Sweden
}

\begin{abstract}
The silver ion does not take up more than two ammonia molecules as ligands in aqueous solutions, even if the solution is saturated with ammonia (J. Bjerrum ${ }^{1}$ ). It was of considerable interest to investigate, whether the diammine silver ion is coordinatively saturated, or if additional ligands other than ammonia, e.g. halide ions, can be coordinated. In this paper mixed complexes of the type $\mathrm{Ag}\left(\mathrm{NH}_{3}\right)_{m} \mathrm{X}_{n}^{1-n}$, are reported where $(m ; n)=(1 ; 1),(1 ; 2)$ and $(2 ; 1)$, if $\mathrm{X}=\mathrm{Cl}$, and $(m ; n)=(1 ; 1),(1 ; 2),(1 ; 3),(2 ; 1)$ and $(2 ; 2)$, if $\mathrm{X}=\mathrm{Br}$. The measurements were performed at $25^{\circ} \mathrm{C}$ and a constant ionic strength of $1 \mathrm{M}$ was maintained by adding ammonium perchlorate.
\end{abstract}

Few investigations of mixed silver ammonia halide complexes have been $\mathbf{F}$ reported. Bodländer and Fittig ${ }^{2}$ determined the solubility of silver chloride in aqueous ammonia and made some potentiometric measurements with silver electrodes. In both cases they found only one complex, viz. $\mathrm{Ag}\left(\mathrm{NH}_{3}\right)_{2}^{+}$. Derr, Stockdale and Vosburgh ${ }^{3}$ measured the same solubility more accurately. They could not describe their measurements quantitatively as simply as Bodländer and Fitting, and they concluded that some mixed complex was also present in their solutions. They did not attempt, however, to identify any such species. Schigol ${ }^{4}$ described his potentiometric measurements by means of the reaction $\mathrm{AgCl}_{2}^{-}+2 \mathrm{NH}_{3} \rightleftharpoons \mathrm{Ag}\left(\mathrm{NH}_{3}\right)_{2} \mathrm{Cl}_{2}^{-}$. The present authors were not able to confirm this particular mixed complex.

\section{THE METHOD}

The solubility of $\mathrm{AgX}$ was determined by shaking the respective halide in a solvent having the composition: $c_{\mathrm{NH}_{3}} \mathrm{MNH}_{3}, c_{\mathrm{NH}_{4} \mathrm{X}} \mathrm{MNH} \mathrm{NH}_{4} \mathrm{X}$ and $\left(l-c_{\mathrm{NH}_{4} \mathrm{X}}\right) \mathrm{M}$ $\mathrm{NH}_{4} \mathrm{ClO}_{4}$. The silver contents of the saturated solutions were determined by electroanalyses for silver chloride and by a tracer technique for silver bromide.

* Present address: Department of Inorganic and Physical Chemistry, University, Lund.

Acta Chem. Scand. 15 (1961) No. 3 
If only mononuclear species are involved, the solid $\mathrm{AgX}$ is dissolved according to the reactions (equilibrium constants $K_{\mathrm{s}, m, n} \mathrm{M}^{2-m-n}$ )

$$
\mathrm{AgX}(\mathrm{s})+m \mathrm{NH}_{3}+(n-1) \mathrm{X}^{-} \rightleftharpoons \mathrm{Ag}\left(\mathrm{NH}_{3}\right)_{m} \mathrm{X}_{n}^{1-n}
$$

Some potentiometric measurements in this laboratory seem to support the view that polynuclear complexes are negligible in the range of concentration pertinent to the measurements of this paper. If $M$ is the maximum number of ammonia molecules that can be attached to one silver ion, and $N$ is the maximum coordination number of silver, the solubility, $l \mathrm{M}$, may be written

$$
l=\sum_{m=0}^{M} \sum_{n=0}^{N-m} \operatorname{Ag}\left(\mathrm{NH}_{3}\right)_{m} \mathrm{X}_{n}^{1-n}=\sum_{m=0}^{M} \sum_{n=0}^{N-m} K_{\mathrm{s}, m, n} a_{\mathrm{NH}_{\mathrm{s}}}^{m}\left[\mathrm{X}^{-}\right]^{n-1}
$$

In practise the summations have to be carried out for values of $m$ and $n$, corresponding to such species that exist in large enough concentrations to have a noticeable influence on the solubility. The total concentration, $l_{\mathbf{x}} \mathrm{M}$, of the pure halide complexes

$$
l_{\mathrm{x}}=\sum_{n=0}^{N}\left[\operatorname{AgX}_{n}^{1-n}\right]
$$

can be determined independently by measuring the solubility of $\mathrm{AgX}$ in solutions free from ammonia. It turned out that $l_{\mathrm{c} 1}$ could be neglected compared with $l$ in the whole range of concentrations used $\left(l_{\mathrm{cl}}<10^{-5}\right.$, cf. also Ref..$\left.^{5}\right)$. On the other hand the solubility of silver bromide in ammonia is influenced by the presence of pure bromide complexes.

To analyse the results the function $A$ is introduced

$$
A=\left(l-l_{\mathrm{x}}\right) \cdot\left[\mathrm{X}^{-}\right] \cdot a_{\mathrm{NH}_{\mathrm{s}}}^{-2}
$$

$A$ would be a constant, $K_{\mathrm{s}, 2,0}$, if no mixed complexes were formed.

Eqns. (2), (3) and (4) may be combined to

$$
A=\sum_{m=1}^{M} \sum_{n=0}^{N-m} K_{\mathrm{s}, m, n} \cdot a_{\mathrm{NH}_{3}}^{m-2} \cdot\left[\mathrm{X}^{-}\right]^{n}
$$

As $m \leqslant 2$ and $m+n \leqslant 4$, and as in the range of our measurements $\left[\mathrm{Ag}\left(\mathrm{NH}_{3}\right)_{2}^{+}\right] \gg\left[\mathrm{Ag}^{+}\right]+\left[\mathrm{AgNH}_{3}^{+}\right]$(vide $\mathrm{J}$. Bjerrum ${ }^{1}$ ), $A$ is in practise simplified to

$$
\begin{gathered}
A=K_{\mathrm{s}, 2,0}+\left(\frac{K_{\mathrm{s}, 1,1}}{a_{\mathrm{NH}_{3}}}+K_{\mathrm{s}, 2,1}\right) \cdot\left[\mathrm{X}^{-}\right]+\left(\frac{K_{\mathrm{s}, 1,2}}{a_{\mathrm{NH}_{3}}}+K_{\mathrm{s}, 2,2}\right) \cdot\left[\mathrm{X}^{-}\right]^{2}+ \\
+\frac{K_{\mathrm{s}, 1,3}}{a_{\mathrm{NH}_{3}}} \cdot\left[\mathrm{X}^{-}\right]^{3}=\sum_{i=0}^{3} B_{i} \cdot\left[\mathrm{X}^{-}\right]^{i}
\end{gathered}
$$

From a series of measurements with almost constant ammonia activity and increasing halide ion concentration, $A$ is obtained as a polynomial function of $\left[\mathrm{X}^{-}\right]$. The coefficients, $B_{i}$, of this polynomial may be computed by the usual graphic methods. If the series is repeated at some other ammonia activities, the equilibrium constants $K_{\mathrm{s}, m, n}$ may be calculated from the values of $B_{i}$ for all such species, as exist in the solutions in sufficient concentrations. This method of calculation has been used for the chloride measurements, where 


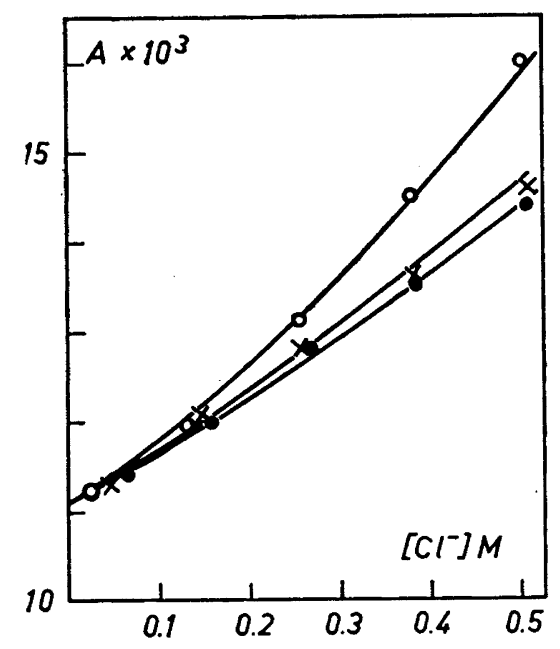

Fig. 1. The function $A\left(\mathrm{Cl}^{-}\right)$. The points are calculated from eqn. (4). $O, \times$ and $O$ denote ammonia activities $0.25,0.5$, and $0.74 \mathrm{M}$, respectively. The curves describe eqn. (6) with the constants from Table 4.

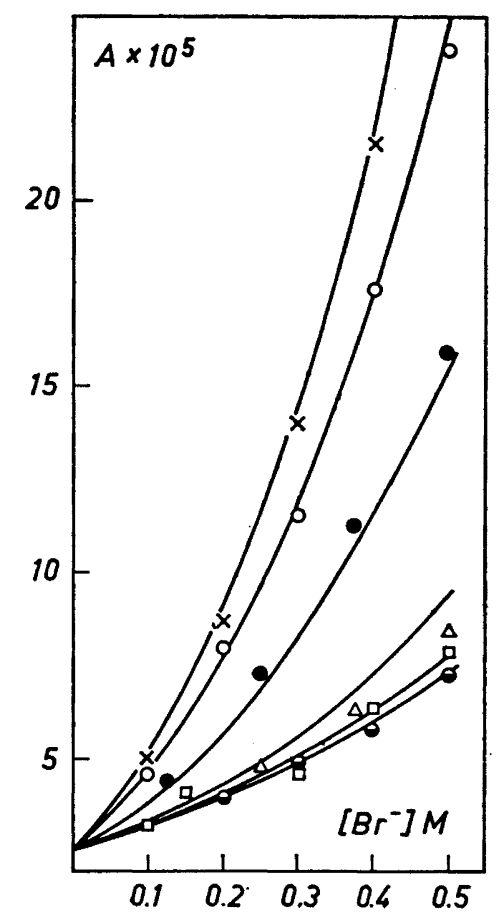

Fig. 2. $A\left(\mathrm{Br}^{-}\right)$. The points are calculated from eqn. (4). $\ominus, \square, \triangle, \odot, O$ and $\times$ denote ammonia concentrations $3.58,3.05,1.80$, $0.70,0.35,0.259 \mathrm{M}$, respectively. The curves represent eqn. (6) with the constants from Table 4.

three equilibrium constants for mixed complexes were enough to describe the results.

In the bromide case the equilibrium constants were computed after a rearrangement of the terms in eqn. (6) in the following way

$$
A=\sum_{i=0}^{2} K_{\mathrm{s}, 2, i} \cdot\left[\mathrm{X}^{-}\right]^{i}+\sum_{i=1}^{3} K_{\mathrm{s}, 1, i} \cdot\left[\mathrm{X}^{-}\right]^{i} \cdot a_{\mathrm{NH}_{3}}^{-1}=A_{0}+A_{1} \cdot a_{\mathrm{NH}_{3}}^{-1}
$$

(i) The family of curves $A\left(\mathrm{X}^{-}\right)$with $a_{\mathrm{NH}_{3}}$ as a parameter representing eqn. (6) is first drawn in a large scale diagram as accurate as possible through the experimental points.

(ii) The curves are cut with lines parallel to the ordinate axis for some values of $\left[\mathrm{X}^{-}\right]$.

(iii) The ordinates for the points of intersection are plotted in a second diagram against $a_{\mathrm{NH}_{3}}^{-1}$, which according to eqn. (7) should result in one straight line for every halide concentration, $\left[\mathrm{X}^{-}\right]$, selected in (ii).

Acta Chem. Scand. 15 (1961) No. 3 


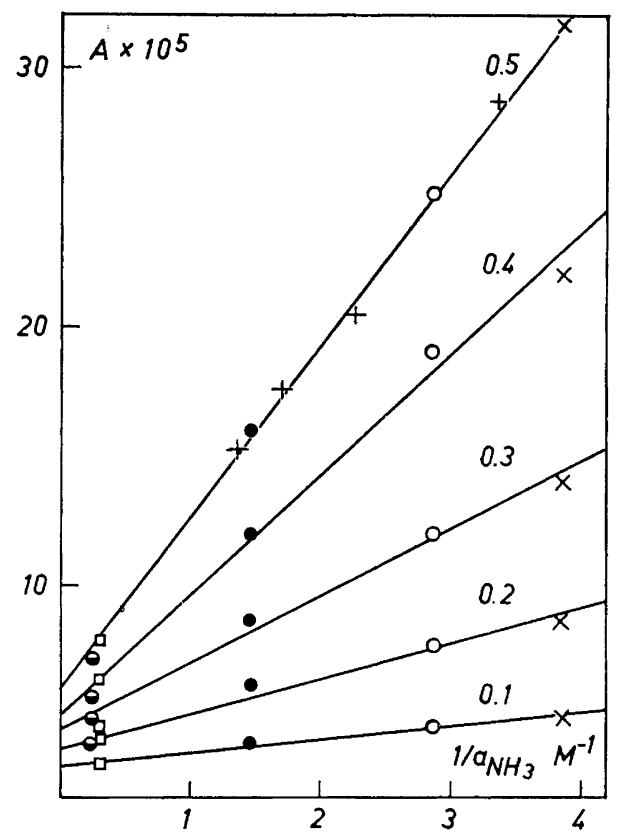

Fig. 3. The lines $A=A_{0}+A_{1} \cdot a_{\mathrm{NH}_{3}}^{-1}$ at the bromide concentrations (M) given at each line. $\ominus, \square, \odot, O$ and $\times$ denote values of $A$, read in Fig. 2 for ammonia activities given there. + denotes values of $A$ from Table 3 .

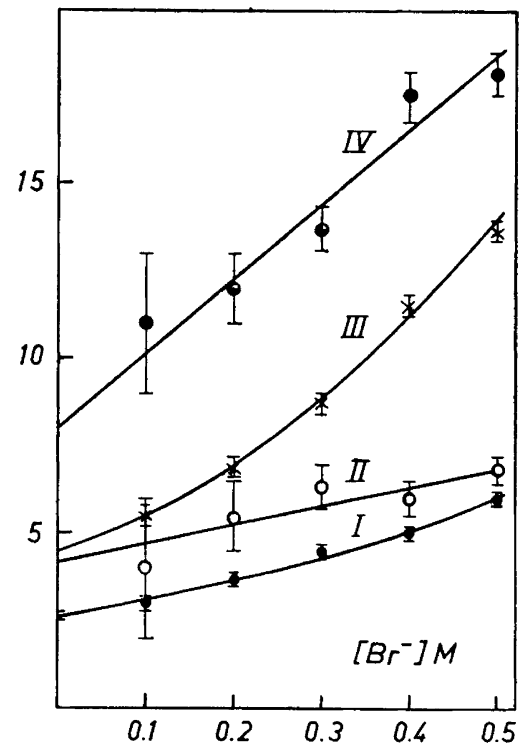

Fig. 4. Computation of the equilibrium constants for the bromide system. The curves are I: $A_{0} \times 10^{5}$; II $\left(A_{0}-2.6\right) \times 10^{5}$ $\left[\mathrm{Br}^{-}\right]^{-1}\left(\mathrm{M}^{-1}\right)$; III: $A_{1} \times 10^{5}\left[\mathrm{Br}^{-}\right]^{-1} ;$ IV: $\left(A_{1} \cdot\left[\mathrm{Br}^{-}\right]^{-1}-4.5\right) \times 10^{5}\left[\mathrm{Br}^{-}\right]^{-1}\left(\mathrm{M}^{-1}\right)$. The plotted values are calculated from the intercepts $\left(A_{0}\right)$ and the slopes $\left(A_{1} \mathrm{M}\right)$ of the lines in Fig. 3.

(iv) The intercept, $A_{0}$, of these straight lines are plotted in a third diagram as a function of $\left[\mathrm{X}^{-}\right]$. From the resulting polynomial curve the equilibrium constants $K_{\mathrm{s}, 2, i}$ are obtained in the usual way, by drawing $\left(A_{0}-K_{\mathrm{s}, 2,0}\right)$ $\left[\mathrm{X}^{-}\right]^{-1}$, which in our case is a straight line with the intercept $K_{\mathrm{s}, 2,1}$ and the slope $K_{\mathrm{s}, 2,2}$.

v) The slopes, $A_{1}$, of the straight lines in (iii) are finally used to calculate $K_{\mathrm{s}, 1, i}$ by plotting $A_{1} \cdot\left[\mathrm{X}^{-}\right]^{-1}$ with $\left[\mathrm{X}^{-}\right]$as the abscissa, which gives $K_{\mathrm{s}, 1,1}$ as the intercept. $K_{\mathrm{s}, 1,2}$ and $K_{\mathrm{s}, 1,3}$ are then obtained as intercept and slope from the straight line $\left(A_{1} \cdot\left[\mathrm{X}^{-}\right]^{-1}-K_{\mathrm{s}, 1,1}\right)\left[\mathrm{X}^{-}\right]^{-1}$ plotted as a function of $\left[\mathrm{X}^{-}\right]$. from

The halide concentrations to be used in the eqns. (2)-(7) were calculated

$$
\left[\mathrm{X}^{-}\right]=c_{\mathrm{NH}_{\mathrm{a}} \mathrm{X}}+l
$$

In only one case was it necessary to correct eqn. (8) for those tiny amounts of halide ions, consumed by the complexes. In this case the concentration of free halide ion is also given (vide Table 1 ). The correction is easily computed by the aid of a preliminary $\bar{n}$-curve (vide e.g. Ref. ${ }^{6}$ ). 
The ammonia activity $a_{\mathrm{NH}_{3}}$ is calculated from

$$
a_{\mathrm{NH}_{8}}=\left(c_{\mathrm{NH}_{3}}-2 l\right) \cdot f_{\mathrm{NH}_{8}}
$$

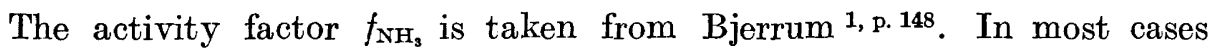
$2 l<<c_{\mathrm{NH}_{3}}$. When this is not the case, the main part of the silver halide is dissolved as species containing two ammonia ligands. Hence, the first factor of the right hand member of eqn. (9) represents the ammonia concentration with sufficient accuracy.

The method of computation described above may be regarded as a special case of a general treatment of similar data given by Sillén ${ }^{7}$ and used by Marcus ${ }^{8}$ for mixed halide mercury complexes.

\section{EXPERIMENTAL DETAILS}

Ammonium perchlorate (Baker's Analyzed), ammonium chloride (Merck's p.a.) and ammonium bromide (The British Drug Houses Ltd) were used without further purification. The stock solutions were analyzed by allowing them to percolate through a hydrogen saturated cation exchanger and then titrating the eluate with sodium hydroxide. No foreign ions could be detected in any of these salts. The ammonia was Merck's p.a. It was standardised by titration with acid. The silver perchlorate used is described elsewhere ${ }^{9}$. The silver chloride was precipitated from hot dilute solutions of silver nitrate and hydrochloric acid. It was carefully washed with water and dried.

The saturation of the solutions with silver halide was carried out in brown or black painted glass bottles with paraffined glass or rubber stoppers. The bottles were rotated in a well controlled thermostat $\left(25 \pm 0.01^{\circ} \mathrm{C}\right)$ for about $20 \mathrm{~h}$. Equilibrium was attained within a tenth of this time. A part $(10-200 \mathrm{ml})$ of the saturated solution was drawn through a glass filter into a pipette. The silver bromide solutions were filtered through a membrane filter (cf. Ref. ${ }^{7}$ ).

The silver chloride solutions were electroanalyzed for silver after some sodium cyanide was added. A few analyses were also carried out by the tracer technique.

The silver bromide was labelled with ${ }^{110} \mathrm{Ag}$ (from Harwell). It was precipitated with ammonium bromide from silver nitrate solutions, prepared by dissolving labelled silver metal in nitric acid. It was thoroughly washed with $1 \mathrm{M} \mathrm{NH} \mathrm{NiO}_{4}$ before use. The activity of the two silver nitrate solutions used were determined as $5.21 \times 10^{8}$ and $1.74 \times 10^{10}$ $\mathrm{ipm} / \mathrm{g}$-atom silver. Their silver contents were determined by electroanalysis and their activity - after dilution with inactive silver nitrate - by means of a scintillation spectrometer (NUKAB, Gothenburg). This apparatus was also used for the solubility determinations.

The solubility determinations were made in the following way. $1.00 \mathrm{ml}$ solution containing $0.1 \mathrm{M}$ inactive silver nitrate and $1 \mathrm{M}$ sodium cyanide was poured into a measuring flask of $10.00 \mathrm{ml}$, which was then filled to the mark with the filtered saturated solution. From this solution $3.00 \mathrm{ml}$ was taken for the activity determination, in which

Table 1. The solubility of silver chloride, $l \mathrm{M}$, and the function $A$.

\begin{tabular}{|c|c|c|c|c|c|c|c|c|c|}
\hline \multirow{2}{*}{$\begin{array}{c}c_{\mathrm{NH}_{3}} \\
(\mathrm{M})\end{array}$} & \multirow{2}{*}{$\begin{array}{c}c_{\mathrm{NH}_{4} \mathrm{Cl}} \\
(\mathrm{M})\end{array}$} & \multirow{2}{*}{$l \times 10^{3}$} & \multirow{2}{*}{$\begin{array}{c}{\left[\mathrm{Cl}^{-}\right]} \\
(\mathrm{M})\end{array}$} & \multirow{2}{*}{$A \times 10^{3}$} & \multirow{2}{*}{$\begin{array}{c}c_{\mathrm{NH}_{4} \mathrm{Cl}} \\
(\mathrm{M})\end{array}$} & \multicolumn{2}{|c|}{$c_{\mathrm{NH}_{3}}=0.494(\mathrm{M})$} & \multicolumn{2}{|c|}{$c_{\mathrm{NH}_{3}}=0.247(\mathrm{M})$} \\
\hline & & & & & & $l \times 10^{3}$ & $A \times 10^{3}$ & $l \times 10^{3}$ & $A \times 10^{3}$ \\
\hline & & & & & & 6.70 & & 1.88 & \\
\hline & & & & & & & & & \\
\hline & & 22 & 0.2 & & 0. & 10. & & & 13 \\
\hline 0.73 & 0.124 & 34. & 0.1 & 12. & 0.125 & 17.9 & 12.1 & 5.13 & 11.9 \\
\hline 0.741 & & 66.2 & 0.064 & 11.4 & & 43.7 & 11.3 & 21.7 & 11.2 \\
\hline
\end{tabular}

Acta Chem. Scand. 15 (1961) No. 3 


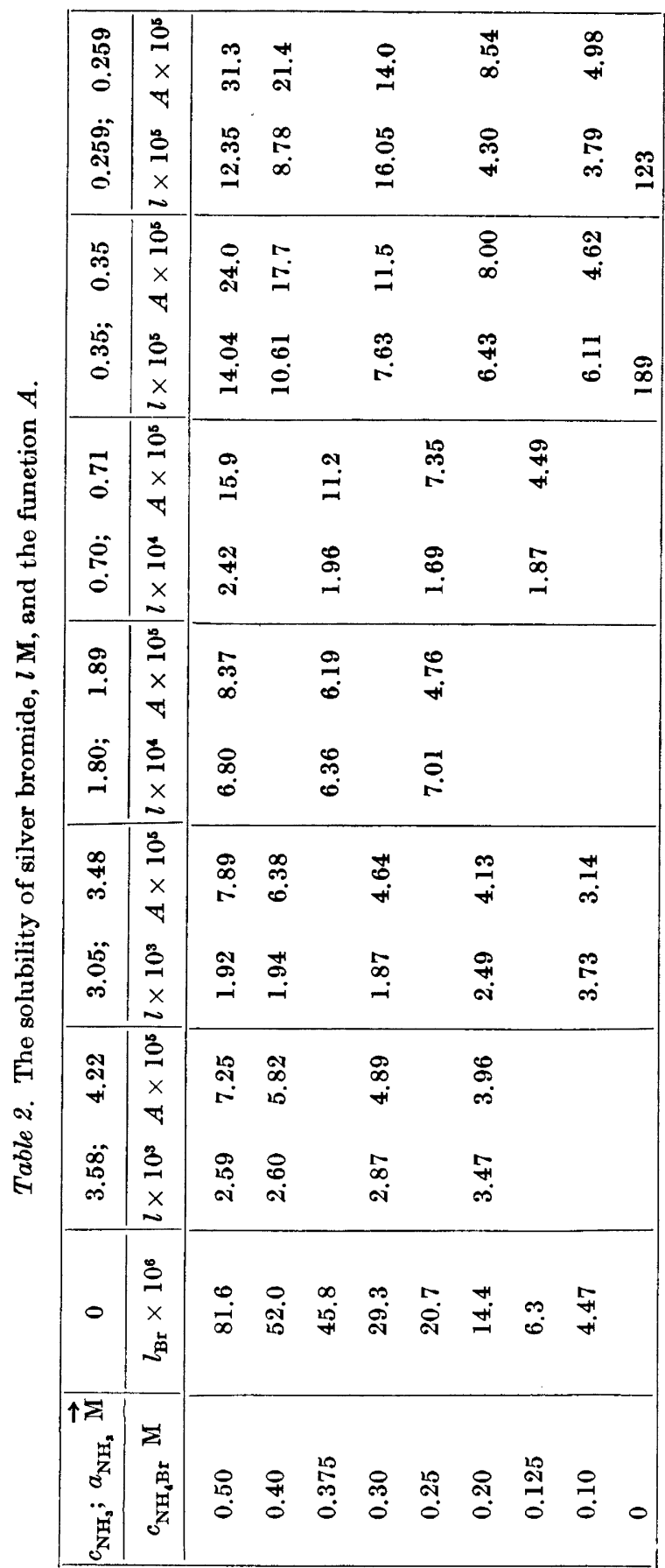

Acta Chem. Scand. 15 (1961) No. 3 
500 to $10^{4}$ impulses were registered. At least 15 such determinations were made for each saturated solution.

The active silver nitrate solutions and the silver bromide were used as quickly as possible after their preparation to avoid difficulties with finely dispersed metallic silver, which was eventually formed because of the radioactivity.

\section{THE SOLUBILITY OF SILVER CHLORIDE}

The results of the chloride measurements are shown in Table 1.

$A\left(\mathrm{Cl}^{-}\right)$is plotted in Fig. 1. For the smallest ammonia concentration $A\left(\mathrm{Cl}^{-}\right)$ is evidently curved, indicating a term of second degree. The curvature is obviously dependent on $a_{\mathrm{NH}_{3}}$. The term $K_{\mathrm{s}, 1,2} / a_{\mathrm{NH}_{3}}$ is essentially as large as the coefficient $B_{2}$, and therefore, $K_{\mathrm{s}, 2,2}$ cannot be determined.

The initial slope of the three lines is only slightly dependent on $a_{\mathrm{NH}_{2}}$. Hence $K_{\mathrm{s}, 2,1}$ predominates in the coefficient $B_{1}$, and the value of $K_{\mathrm{s}, 1,1}$ becomes very uncertain. The curves are drawn with the constants $K_{\mathrm{s}, m, n}$ of Table 4 $\left(B_{3}=K_{\mathrm{s}, 2,2}=0\right)$.

\section{THE SOLUBILITY OF SILVER BROMIDE}

The solubility of silver bromide is shown in Tables 2 and 3 . The values $A\left(\mathrm{Br}^{-}\right)$from Table 2 are plotted in Fig. 2. The intercepts between the curves $A\left(\mathrm{Br}^{-}\right)$and five vertical lines, $\left[\mathrm{Br}^{-}\right]=0.1,0.2,0.3,0.4$, and $0.5(\mathrm{M})$, are plotted against $a_{\mathrm{NH}_{3}}^{-1}$ in Fig. 3., in which each value of [Br $\left.{ }^{-}\right]$from Fig. 2 gives one straight line. For $\left[\mathrm{Br}^{-}\right]=0.5(\mathrm{M})$ the values of $A$ from Table 3 are also plotted in Fig. 3. Finally the equilibrium constants, $K_{\mathrm{s}, m, n}$, are obtained from $A_{0}$ and $A_{1}$ by the usual graphical methods as shown in Fig. 4. These constants have been used when drawing the curves in Fig. 2. The experimental points lie on these curves within fair accuracy. Hence the presented scheme describes the solubility over the range of concentrations, $a_{\mathrm{NH}_{3}}<4(\mathrm{M})$ and $\left[\mathrm{Br}^{-}\right]<0.5$ (M), covered by the experiments. Of course, some of the equilibrium constants can only be obtained with a rather small accuracy (vide Table 4), viz. those constants corresponding to complexes whose concentrations never exceed a

Table 3. The solubility of silver bromide, $l \mathrm{M}$, at variated ammonia concentrations, $c_{\mathrm{NH}_{3}} \mathrm{M}$, in $1 \mathrm{M} \mathrm{NH}_{4} \mathrm{ClO}_{4}$ and in $0.5 \mathrm{M} \mathrm{NH}_{4} \mathrm{Br}+0.5 \mathrm{M} \mathrm{NH}_{4} \mathrm{ClO}_{4}$

\begin{tabular}{|l|c|c|c|c|c|}
\hline \multicolumn{3}{|c|}{$c_{\mathrm{NH}_{6} \mathrm{Br}}=0$} & \multicolumn{3}{|c|}{$c_{\mathrm{NH}_{6} \mathrm{Br}}=0.5(\mathrm{M})$} \\
\hline \multicolumn{2}{|c|}{$c_{\mathrm{NH}_{3}}$} & \multicolumn{2}{|c|}{$l \times 10^{4}$} & $A \times 10^{5 * * *}$ & \multicolumn{3}{c|}{$c_{\mathrm{NH}_{3}}$} & $l \times 10^{5}$ & $A \times 10^{5}$ \\
\hline & & & & & \\
$6.20^{*}$ & 449 & 2.62 & 0.739 & 24.9 & 15.3 \\
$2.07 * *$ & 117 & 2.73 & 0.591 & 20.5 & 17.6 \\
0.61 & 31.2 & 2.62 & 0.443 & $16.2_{2}$ & 20.5 \\
0.366 & 18.8 & 2.64 & 0.296 & $13.1_{6}$ & 28.7 \\
0.244 & 12.5 & 2.61 & 0.148 & $10.2_{7}$ & 48.3 \\
0.122 & 6.3 & 2.67 & 0 & $8.1_{6}$ & - \\
\hline
\end{tabular}

* $a_{\mathrm{NH}_{3}}=8.74 . \quad * * a_{\mathrm{NH}_{3}}=2.21 . \quad * * *$ Mean of $A=2.6_{3} \times 10^{-5}=K_{\mathrm{s}, 2,0}$

Acta Chem. Scand. 15 (1961) No. 3 
Table 4. The equilibrium constants, $K_{\mathrm{s}, m, n} \mathrm{M}^{2-m-n}$, for silver chloride (left column) and silver bromide (right column).

\begin{tabular}{|c|c|c|}
\hline$m, n$ & $K_{\mathrm{s}, m, n} \times 10^{3}$ & $K_{\mathrm{s}, m, n} \times 10^{5}$ \\
\hline 2,0 & $11.1 \pm 0.1$ & 2.6 .0 .05 \\
2,1 & $5 \pm 1$ & $4.3 \pm 0.5$ \\
2,2 & & $5 \pm 2$ \\
1,1 & $0.4 \pm 0.4$ & $4.5 \pm 0.5$ \\
1,2 & $1.5 \pm 0.5$ & $8 \pm \pm 4$ \\
1,3 & & $20 \pm 10$ \\
\hline
\end{tabular}

rather small part of the total silver concentration in the solution. The possible errors, also given in Table 4, can be estimated rather well from the trials to draw the "best" curves through the experimental points in Fig. 4.

\section{THE EQUILIBRIUM CONSTANTS}

From the constants in Table 4 the distribution of silver on the different species can easily be computed. The fraction $\alpha_{m, n}$ of silver, bound in $\mathrm{Ag}\left(\mathrm{NH}_{3}\right)_{m} \mathrm{X}_{n}^{1-n}$, is

$$
\alpha_{m, n}=\frac{K_{\mathrm{s}, m, n} \cdot a_{\mathrm{NH}_{\mathrm{s}}}^{m} \cdot\left[\mathrm{X}^{-}\right]^{n-1}}{l}
$$

where $l$ is obtained from eqn. (2) in which all constants are now known. It follows that $\mathrm{Ag}\left(\mathrm{NH}_{3}\right)_{2}^{+}$is highly predominant in the chloride solutions, since it never contains less than about two thirds of the total silver in the solutions within the range studied. In the bromide solutions, however, the silver is rather evenly distributed on several species at the highest bromide concentrations.

For the calculation of the gross complexity constants the solubility products of silver chloride and bromide must be known in $1 \mathrm{M} \mathrm{NH}_{4} \mathrm{ClO}_{4}$. These constants together with the constants for the stepwise formation of the complexes shall be discussed further elsewhere in connection with some potentiometric measurements in unsaturated solutions of silver halides.

Acknowledgements. Our thanks are due to Professor Cyrill Brosset, who put the facilities of his laboratory to our disposal for this work, and to Dr. Eric Berne for valuable advice for the tracer measurements. The English of this paper has been corrected by Dimitrios Karipides, Ph.D., Pennsylvania State University.

\section{REFERENCES}

1. Bjerrum, J. Metal Ammine Formation in Aqueous Solution (Diss.) Copenhagen 1941.

2. Bodländer, G. and Fittig, R. Z. physik. Chem. 39 (1902) 597.

3. Derr, P. F., Stockdale, R. M. and Vosburgh, W. C. J. Am. Chem. Soc. 63 (1941) 2670.

4. Schigol, M. B. Zhur. Obschei Khim. 22 (1952) 723.

5. Berne, E. and Leden, I. Svensk Kem. Tidskr. 65 (1953) 88.

6. Leden, I. Acta Chem. Scand. 10 (1956) 812.

7. Sillén, I. G. Acta Chem. Scand. 10 (1956) 803.

8. Marcus, Y. Acta Chem. Scand. 11 (1957) 811.

9. Leden, I. Svensk Kem. Tidskr. 64 (1952) 249.

Received November 17, 1960.

Acta Chem. Scand. 15 (1961) No. 3 